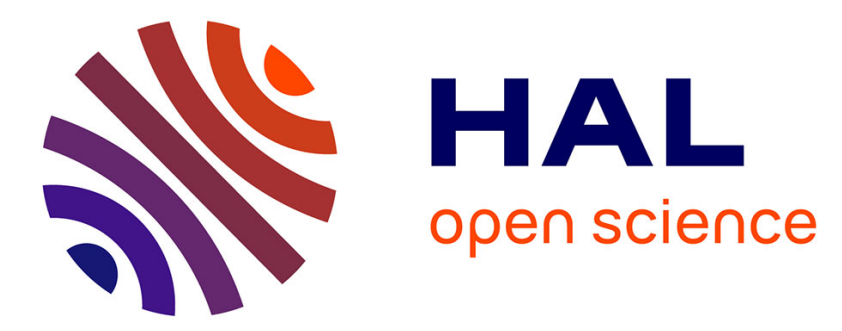

\title{
CCA Threshold Impact on the MAC Layer Performance in IoT Networks
}

\author{
Abderrahman Ben Khalifa, Razvan Stanica
}

\section{To cite this version:}

Abderrahman Ben Khalifa, Razvan Stanica. CCA Threshold Impact on the MAC Layer Performance in IoT Networks. VTC Spring 2020 - IEEE 91st Vehicular Technology Conference, May 2020, Antwerp,

Belgium. hal-02878723

\section{HAL Id: hal-02878723 \\ https://hal.inria.fr/hal-02878723}

Submitted on 23 Jun 2020

HAL is a multi-disciplinary open access archive for the deposit and dissemination of scientific research documents, whether they are published or not. The documents may come from teaching and research institutions in France or abroad, or from public or private research centers.
L'archive ouverte pluridisciplinaire HAL, est destinée au dépôt et à la diffusion de documents scientifiques de niveau recherche, publiés ou non, émanant des établissements d'enseignement et de recherche français ou étrangers, des laboratoires publics ou privés. 


\title{
CCA Threshold Impact on the MAC Layer Performance in IoT Networks
}

\author{
Abderrahman Ben Khalifa, Razvan Stanica \\ Univ Lyon, INSA Lyon, Inria, CITI, F-69621 Villeurbanne, France \\ Email: abderrahman.ben-khalifa@insa-lyon.fr
}

\begin{abstract}
While current medium access control solutions in low-power wide area networks are generally based on Aloha, recent studies demonstrated the interest of adding carrier sense mechanisms to the picture. In this paper, we investigate the impact of the carrier sense threshold parameter in this particular context. We show that its impact on the average behavior of the network is limited, but this changes when looking at the individual node performance. Our simulation results demonstrate an important heterogeneity among nodes, both in terms of packet success probability and of energy consumption. Moreover, the performance of the nodes is strongly correlated with the percentage of contending nodes that they can sense. By simply using two different carrier sense thresholds in the network, we achieve an increased fairness among nodes.
\end{abstract}

\section{INTRODUCTION}

With an increasing appetite in our society for connected objects, current wireless technologies need to be adapted to the context of the Internet of Things (IoT). With very specific properties, such as high node density and important energy constraints, the IoT use case produced a significant number of dedicated technologies, currently competing on the market, such as Sigfox [1], LoRa [2], NB-IoT [3], or WiFi HaLow [4], to cite just a few.

Most of these technologies are using Aloha as a medium access control (MAC) layer protocol, which is practically the most basic solution in the field. This choice can be explained by the fact that Aloha is easy to implement and consumes only a small amount of energy. Moreover, dedicated IoT networks present an important asymmetry between the base station and the connected objects. Indeed, the electronic part of the latter is much simpler than the one of the former, meaning that the objects have a much lower receiver quality when compared to the base station. This means that an object can not reasonably be expected to sense all the other objects in the communication range of the base station, resulting in the well known hidden terminal problem [5], which highly degrades the performance of carrier sense medium access (CSMA) solutions.

However, recent studies argue that including a carrier sense mechanism in low-power wide area networks (LPWAN) can bring important benefits in terms of reliability, with an energy cost that remains sustainable [6], [7]. These gains are achieved even when the carrier sense range of the objects is much lower than the one of the base stations (hundreds of meters vs. kilometers in [7]).

If solutions based on carrier sense are to be used in dedicated IoT scenarios, an essential parameter is the clear channel assignment (CCA) threshold. Practically, the lower the CCA threshold of a node, the larger the area it can sense. The impact of the CCA threshold in networks using CSMA with collision avoidance (CSMA/CA) has been investigated in different contexts: wireless local area networks [8], mobile ad-hoc networks [9], or vehicular networks [10]. However, as far as our knowledge goes, the impact of the carrier sense mechanism was never considered in an IoT context, with its specific properties. In this paper, we conduct a detailed simulation study of a dedicated IoT network, varying the CCA threshold of the nodes. This has a double interest in our case. First of all, it allows us to study a realistic IoT network, where the carrier sense range of the nodes is reduced with respect to the one of the IoT base station. Second, it permits us to test, for the first time in the literature, the impact of a heterogeneous CCA threshold in these networks.

We articulate this paper around four contributions. First, we show that the CCA threshold has only a very limited impact on the packet success probability (PSP) and on the energy consumption of the IoT nodes, a different behaviour with respect to other types of networks [8], [9], [10]. Second, we define a new metric, denoted as the CCA conflict rate, which embeds information regarding the percentage of contending nodes that a connected object can sense when its CCA mechanism is active. Third, by observing the individual node behavior, we notice significant differences between nodes, with individual performance highly correlated to the CCA conflict rate. Finally, we show that fairness among nodes can be increased by simply using two different CCA threshold values in the network.

We begin with a brief discussion of related work in Sec. II, before descibing our simulation study in Sec. III. The impact of the CCA threshold on the average behavior of the network is investigated in Sec. IV. The CCA conflict rate is defined and studied in Sec. V. Finally, the use of heterogeneous CCA threshold in an IoT network is evaluated in Sec. VI, before concluding remarks in Sec. VII.

\section{RELATED WORK}

While most dedicated IoT technologies are based on Aloha solutions at the MAC layer, WiFi HaLow, standardized as IEEE 802.11ah [11], natively uses CSMA/CA. As all classical WiFi solutions, IEEE 802.11ah does not allow the adaptation of the CCA threshold. However, this static assignment is bound to change with the development of the IEEE 802.11ax 
technology [12], also known as WiFi 6 and recently available on the market, where the major novelty is dynamic receiver sensitivity.

Recent studies also challenge the idea that LPWAN solutions such as LoRa or Sigfox do not need a carrier sense mechanism. Pham [7] implemented different carrier sense solutions on LoRa hardware, showing improved performance in the case of image sensing IoT devices. Using a simulation approach, To and Duda [6] demonstrate similar properties. Moreover, they show that using a non-persistent CSMA approach results in an energy consumption close to the one obtained by Aloha. In our previous work [13], we also looked at the impact of CSMA/CA in dedicated IoT networks, showing a significant increase in terms of packet reception ratio in dense networks, at a price of a much higher energy consumption (unlike [6], we tested a persistent CSMA approach, which consumes much more energy). However, [7] only considers a lightly loaded network, while [6] and [13] consider that the nodes have a very large carrier sense range, equivalent to the one of the IoT base station, an assumption we challenge in this work.

The impact of the CCA threshold is fairly well understood in IEEE 802.11 networks [8], [9], [10], [14]. Basically, by increasing the CCA threshold, a node becomes less sensitive to surrounding transmissions, decreasing its carrier sense range. This increases the spatial reuse in the network, while also increasing the number of hidden terminals. An optimal CCA threshold, maximizing the network throughput, can be found, depending on the network density and traffic intensity. However, the IoT context is different from these previous studies, in terms of traffic model and metrics of ineterest.

\section{Simulation Methodology}

We use the Network Simulator 3 (ns3) [15] to study a dedicated IoT network with $N$ sender nodes and one sink node. The most important simulation parameters are provided in Tab. I. While we provide these values for reproducibility purposes, we argue that our study is agnostic to the physical layer parameters and models. We acknowledge that, because of this choice, our findings do not directly apply to a specific IoT technology. Indeed, our intention is to be as generic as possible and study a dedicated IoT network based on its particular topology and traffic model, not on the specific functions it implements at the physical layer.

Basically, we set our simulation in order to obtain a network topology consisting of a single cell of radius $r$, with a base station sink node situated in its center. The IoT nodes are uniformly distributed inside this area and they all share the same channel. Each sender node produces one packet of data each time period $T$, while the sink node only transmits ACK frames. Different IoT technologies achieve very different data rates at the physical layer, from $100 \mathrm{~b} / \mathrm{s}$ in Sigfox to several $\mathrm{Mb} / \mathrm{s}$ in WiFi HaLow. In order to have a fair, but technology agnostic comparison, we use as a parameter the transmission opportunity, $T_{o p}=S / T$, where $S$ is the airtime of a MAC layer frame. As an example, a $T_{o p}$ value of $165 \cdot 10^{-6}$ corresponds to a packet arrival every second in WiFi HaLow and every 20 minutes in Sigfox.

We use performance metrics that we consider relevant for the IoT context: the PSP and the time duration spent by each node in an ON state (receiving, transmitting or listening to the channel). We believe that this second metric is a good generic proxy for the energy consumption of a node and we use it instead of a more classical metric, which would once again require the use of technology-specific parameters.

\begin{tabular}{|c||c|}
\hline \multicolumn{1}{|c||}{ Parameter } & Value \\
\hline Acknowledgement Timeout & $75 \mathrm{~ms}$ \\
\hline Maximum Number of Retransmission & 7 \\
\hline RTS/CTS message exchange & Disabled \\
\hline Frequency & $5.180 \mathrm{GHz}$ \\
\hline Transmission Power & $16 \mathrm{dBm}$ \\
\hline Propagation Loss Model & Log Distance \\
\hline Propagation Delay Model & Constant Speed Mode \\
\hline Transmission Data Rate & $6 \mathrm{Mbps}$ \\
\hline Transmission Opportunity & $165 \cdot 10^{-6}$ \\
\hline
\end{tabular}

TABLE I

DEFAULT SIMULATION PARAMETERS.

For the CSMA/CA protocol, we used the ns3 AdhocWifi$\mathrm{Mac}$ as a MAC layer model, using the parameters indicated in Tab. I. The Clear Channel Assessment (CCA) threshold is set by default at $-99 \mathrm{dBm}$. However, as explained below, we studied different values for this parameter in our study. Another parameter that we observe in detail is the maximum number of retransmissions used by the CSMA/CA protocol. Indeed, if the reception of a message is not confirmed by an acknowledgment message, the CSMA/CA MAC layer retransmits the message in question. This is essential in the case of a file download, for example, where the integrity of the content needs to be ensured. However, IoT applications are often less sensitive to packet losses, and limiting the number of authorized retransmissions can actually reduce the channel contention. This is why we show results for two values of maximum allowed retransmissions, denoted as $M: 3$ and 7 .

We run simulations while varying the number of sender nodes. Every simulation lasts 30 seconds and it is repeated 10 times, with a different seed value each time. All the results presented in the remainder of the paper that show average values are given with a confidence interval of $95 \%$.

\section{CCA THRESHOLD IMPACT}

In this section, we study the effect of the CCA threshold on the CSMA/CA protocol in an IoT context, by evaluating the PSP and the time spent by the nodes in an active state.

\section{A. Packet Success Probability}

The essential performance metric in IoT networks is the success probability of a message. The small size of the messages transmitted by the nodes allows their encapsulation into single packets, which are possibly transmitted multiple times as MAC layer frames. Since we are interested in the overall performance of the MAC layer, in Fig. 1 we present the average packet success probability of the CSMA/CA protocol, 


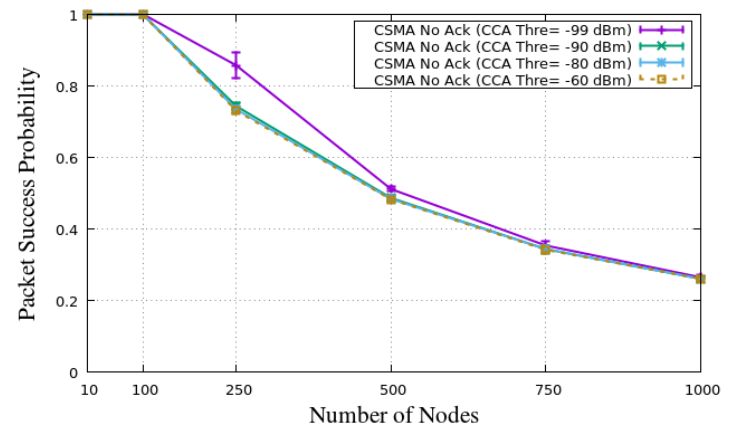

(a) Maximum Transmission Number set at 7.

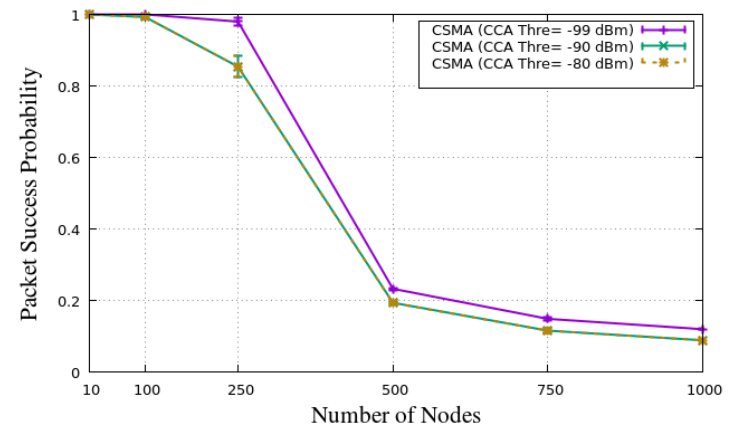

(b) Maximum Transmission Number set at 3.

Fig. 1. CSMA/CA Packet Success Ratio for different CCA Threshold values.

calculated for different numbers of contending nodes and different values of the CCA threshold.

In Fig. 1a, presenting results for $M=7$, we can see that all the curves are showing the same shape: the global success probability tends to decrease for networks with higher density and the CCA threshold does not have a significant impact on the global packet success probability of the network. In Fig. $1 \mathrm{~b}$, where $M=3$, the similarity of the curves shapes is still present. In this case, we see that the lowest CCA threshold value gives a slightly better performance for all network densities than the higher CCA threshold values.

\section{B. Activity Time}

The energy consumption is an important metric for most IoT devices, constrained in terms of size, hence battery. In this work, we do not directly compute the node energy consumption, as this would require a technology-specific energy model. Instead, we calculate a correlated metric, the duration each node spends in an active $\mathrm{ON}$ state, i.e. the time the node is using its radio module, either for transmission, reception or listening the channel. Since we study a CSMA/CA access scheme, we consider that the node is active in order to sense the channel during the back-off slots, as well as shortly after the transmission, when it is waiting for an ACK message.

As shown in Fig. 2a, for $M=7$, we see that changing the CCA threshold is not having any impact on the node activity time. In Fig. $2 \mathrm{~b}$, for $M=3$, we see that, for a medium density network, a CCA threshold of $-99 \mathrm{dBm}$ is more economic than the other values, which are all presenting similar behaviours.

It is curious to notice that modifying such an important MAC layer parameter (i.e. the maximum number of retransmissions) is not having a major impact on the global network performance. Another important remark is that, in some cases, MAC solutions with a higher CCA threshold are providing worse performance in terms of success probability, while consuming as much energy as lower CCA threshold values. For our IoT context, where the nodes are devices with limited power resources, it is interesting to discover that, by changing the value of one MAC layer parameter, we can have the same transmission reliability, while paying a lower energy cost.

\section{CCA Conflict RATe}

The results in the previous section, as well as most of the results in the literature, consider that the nodes have a large carrier sense range, covering all of their contenders for channel access. As explained, this assumption is particularly unrealistic in a dedicated IoT scenario, where the nodes have much cheaper and simpler electronics compared to an IoT base station, resulting in a reduced receiver sensitivity. We therefore argue that it is not realistic to consider that a node senses all the other nodes in the cell. By changing the CCA threshold of the nodes, we can model this phenomenon, where only a part of the transmissions towards the base station can be sensed by the nodes. Indeed, depending on their position in the network and on their CCA threshold, nodes can have a certain number of hidden interferers, a phenomenon well known in the literature [16].

To assess the impact of the hidden terminals in our network, we define a metric denoted as the CCA conflict rate. This metric is calculated for each node and it shows the ratio of nodes in the cell with which the considered node is in contention, in other words the rate of nodes in the cell that are in the CCA detection zone of the considered node. This parameter depends directly on the value of the CCA threshold and it is calculated for a node $A$ as follow:

$$
C C A \_C R_{A}=\frac{1}{(N-1)} \sum_{i \in N \backslash\{A\}} \frac{R x_{i}^{A}}{T x_{i}}
$$

where $R x_{i}^{A}$ represents the number of frames overheard by node $A$ from the total $T x_{i}$ frames transmitted by node $i$. Our goal is to measure the number of neighbors of node $A$, while also accounting for the probabilistic nature of the radio propagation model. Indeed, in some cases, only a part of the messages transmitted by node $i$ actually activate the carrier sense mechanism of node $A$, and this definition allows accounting for these situations.

In this section we study the CCA conflict rate of each node and verify whether this metric is linked to the node packet success probability and energy consumption. For this, in our simulations we calculate the CCA conflict of each node for different CCA thresholds and network densities. 


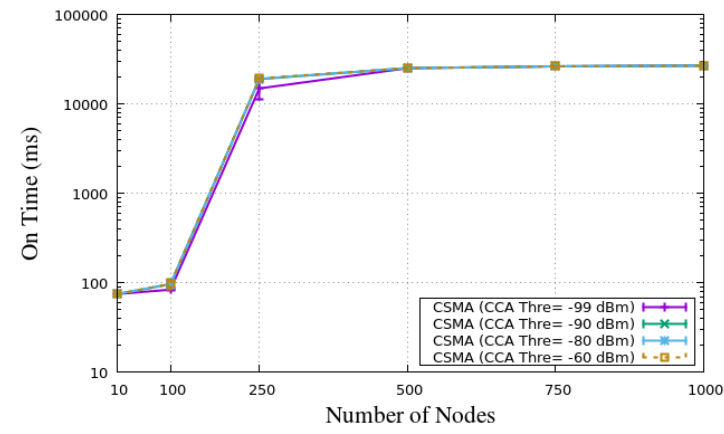

(a) Maximum Transmission Number set at 7 .

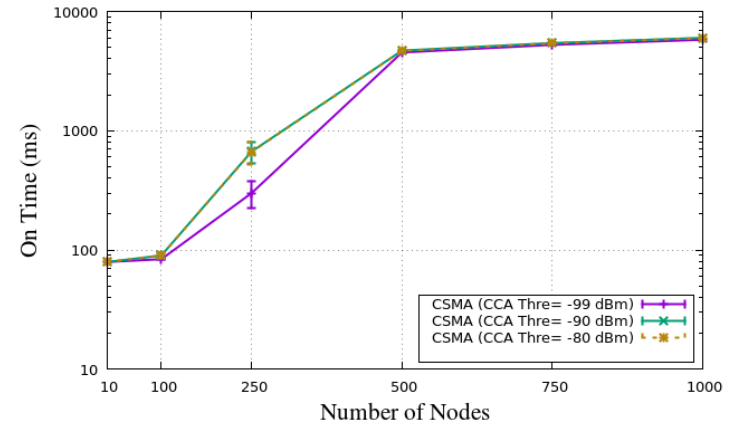

(b) Maximum Transmission Number set at 3 .

Fig. 2. CSMA ON Time (ms) for different CCA Threshold values.

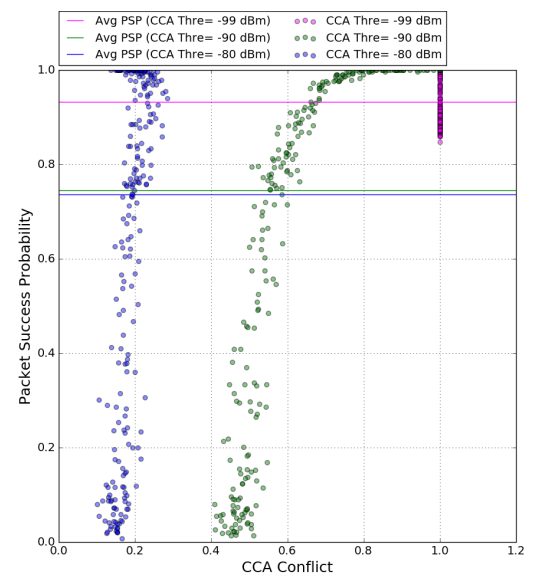

(a) A network of 250 nodes.

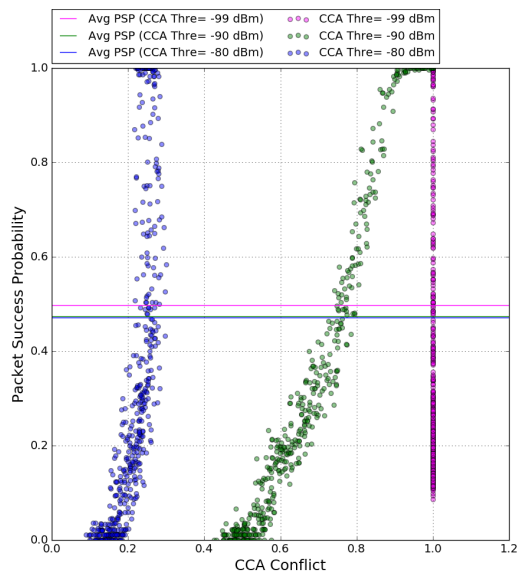

(b) A network of 500 nodes.

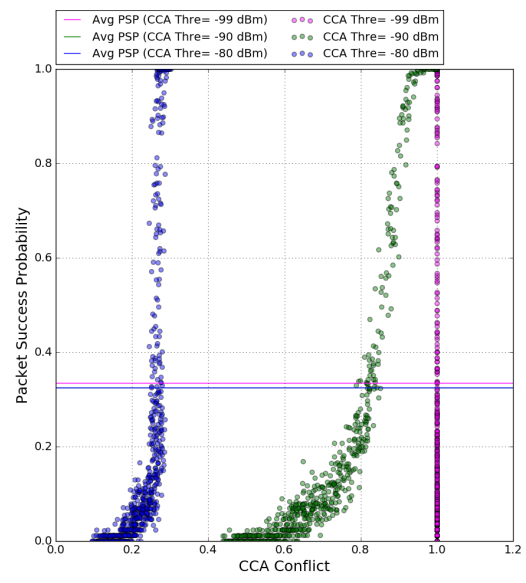

(c) A network of 750 nodes.

Fig. 3. Nodes PSP and CCA Conflict for different CCA Threshold values

\section{A. Packet Success Probability}

In Fig. 3, we show the node packet success probability and its CCA conflict rate for three different network sizes: 250 nodes, 500 nodes and 750 nodes. As expected, when the CCA threshold is set at $-99 \mathrm{dBm}$, the CCA conflict rate is equal to 1 for all the nodes. This means there is one CCA detection zone, common to all nodes: each node in the cell is capable to sense all the other nodes, in a perfect clique. Of course, it is normal not to detect any correlation between the CCA conflict rate and the PSP of the nodes in such a situation.

However, when we increase the CCA threshold at a value of $-90 \mathrm{dBm}$, we see a clear correlation between the two axis and we notice that the nodes with a higher CCA conflict rate are the ones getting a better PSP result and vice versa, and this is true for all the network densities. If we further increase the CCA threshold at $-80 \mathrm{dBm}$, we still see the correlation, but not as clear as for $-90 \mathrm{dBm}$, and on a thinner interval. We believe that this correlation will disappear if we continue increasing the CCA threshold until we reach a CCA conflict rate of zero for all the nodes, which is supposed to be the case of a network using the Aloha protocol at the MAC layer.

In Fig. 3a, we show the case of a relatively low traffic network of 250 nodes. This is the only situation where we observe a significant change of the average PSP when modifying the CCA threshold. More precisely, the lowest value of the CCA threshold is getting $10 \%$ better PSP than the two other solutions $(-90 \mathrm{dBm}$ and $-80 \mathrm{dBm})$. Observing individual node performance in the same figure, reducing the CCA threshold is having a remarkable effect, with all the nodes reaching a PSP higher than $82 \%$, while for the two other CCA thresholds we have almost one third of the nodes that are getting a PSP lower than $75 \%$ and $8 \%$ of the nodes with less than $20 \%$.

In Fig. 3b, showing a network of 500 nodes, we see that the average PSP is not changing significantly with the variation of the CCA threshold (2.5\% difference between the three threshold values). On the other hand, looking at the individual node performance, when a CCA threshold of $-99 \mathrm{dBm}$ is used, all the nodes in the cell obtain a PSP higher than $10 \%$. Meanwhile, the two other CCA thresholds result in $20 \%$ of the nodes with a PSP less than $10 \%$, and $5 \%$ of the nodes with a PSP near zero. For a denser network of 750 nodes, depicted in Fig. 3c, we barely see any difference for the average PSP result between the different CCA thresholds. And, even for the local node performance, we do not notice any significant change 


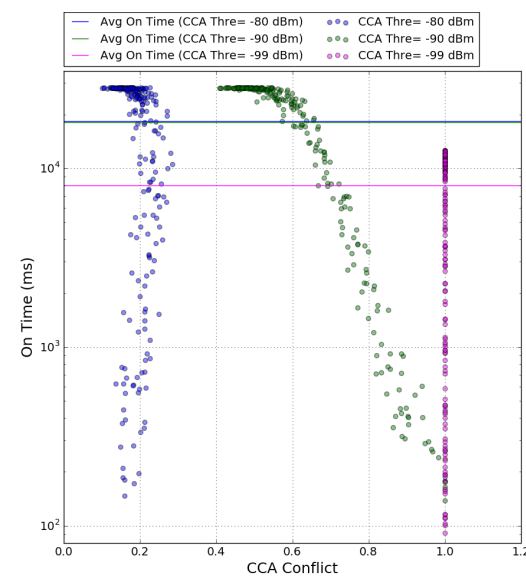

(a) A network of 250 nodes.

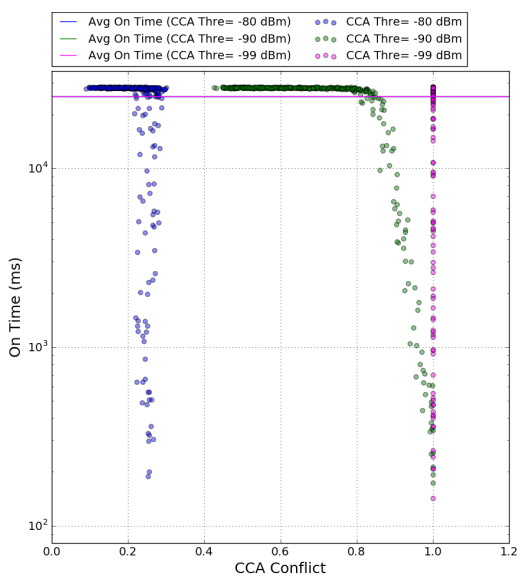

(b) A network of 500 nodes.

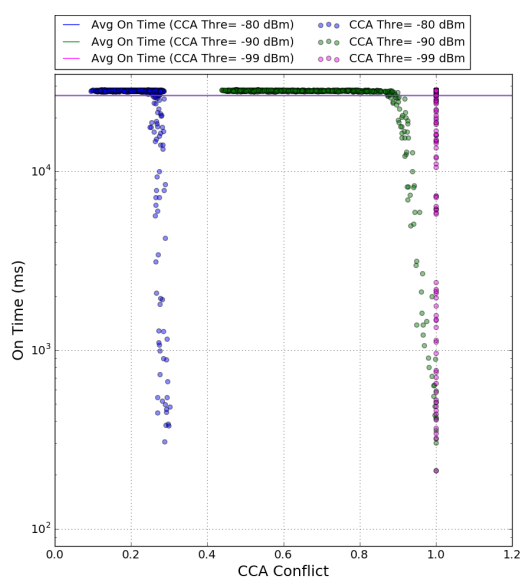

(c) A network of 750 nodes.

Fig. 4. Nodes Energy Consumption and CCA Conflict for different CCA Threshold values

when we modify the CCA threshold value. We believe that, in such high density conditions, the carrier sense mechanism reaches its limits and it is no longer sufficient in the fight against the inherent collisions.

For these results, obtained with $M=7$, we can conclude that the CCA threshold has a limited impact on the global performance of an IoT network, and this only under a low network density. However, the results at an individual node level show an important heterogeneity, with nodes reaching a reception ratio close to $100 \%$, while others are practically disconnected from the base station. Fine tuning the CCA threshold can alleviate this problem up to a certain point, reducing the number of completely disconnected nodes. Of course, when the network density becomes too important, the limits of the carrier sense mechanism are attained.

\section{B. Node Activity Time}

Fig. 4 shows the activity time of each node, as well as their CCA conflict rate. We can easily notice that the energy consumption is behaving the same way as the PSP with respect to the CCA threshold. For a CCA threshold set at $-99 \mathrm{dBm}$, the CCA conflict rate is equal to 1 for all the nodes; as a consequence, no correlation can be observed between the two axis. However, this correlation is present for a CCA threshold of $-90 \mathrm{dBm}$ and, at a smaller scale, at $-80 \mathrm{dBm}$ as well. This result is due to the fact that the correlation disappears when we highly increase the CCA threshold and we get closer to an Aloha behaviour, as explained in the previous section.

It is important to note the particularity of the correlation in the case of the node activity time: below a certain CCA conflict rate limit, the activity time becomes almost constant and independent of the CCA conflict rate. Above this limit, the correlation is clear and the higher the CCA conflict rate of a node, the higher its energy saving. This is because nodes with higher CCA conflict rates better avoid collisions, which results in less retransmissions, hence in less consumed energy.
The only impact of the CCA threshold on the average node activity time is observed for a relatively low dense network of 250 nodes, as seen in Fig. 4a. The global energy consumption with a CCA threshold of $-99 \mathrm{dBm}$ is significantly lower than in the two other settings. For the denser networks, with 500 and 750 nodes, no difference was found regarding the node activity time under different CCA threshold values. Hence, we can conclude that, for highly dense networks, the energy consumption tends to be independent of the CCA threshold even if, for higher values of this parameter, the node spends less time sensing the channel in back-off mode and transmit faster. This independence can be explained by the presence of a second parameter, namely the contention window of the backoff mechanism, which has a dominant impact on the energy consumption in high traffic situations.

However, as for the reception ratio, we remark that the global behaviour is not reflected at a local node level. Indeed, some nodes are consuming much more energy than others, with differences that can reach two orders of magnitude. Moreover, further investigations show that these high consuming nodes are the same with a low success probability in Fig. 3.

\section{Heterogeneous CCA Threshold}

In this section, we start from the observation that the fairness in an IoT network is very low. While some nodes are well placed in the network, with a very high packet success rate and low energy consumption, others are not only practically disconnected from the base station, but they are also consuming a much larger amount of energy. Therefore, we investigate the consequences of using different CCA threshold values within the network, specially in the performance region where the PSP is sensitive to the CCA threshold change. We note that we do not propose an adaptive CCA threshold mechanism, but we simply evaluate the consequences of using nodes with heterogeneous CCA threshold settings.

For the case of a network of 500 nodes and a CCA threshold of $-90 \mathrm{dBm}$ and with $M=7$ we saw, in Fig. $3 \mathrm{~b}$, that the 


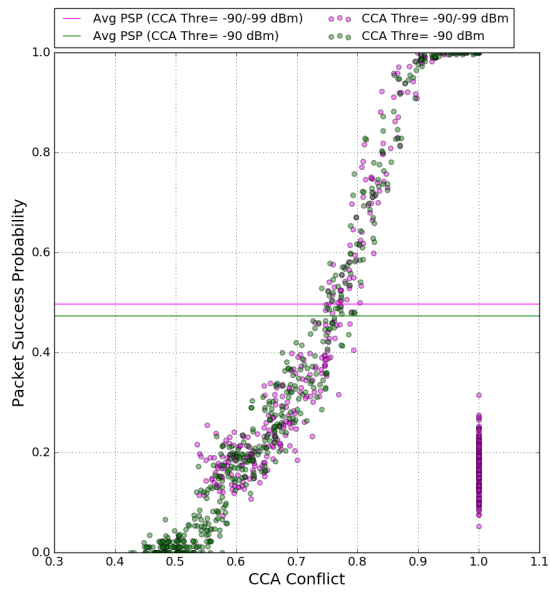

(a) PSP.

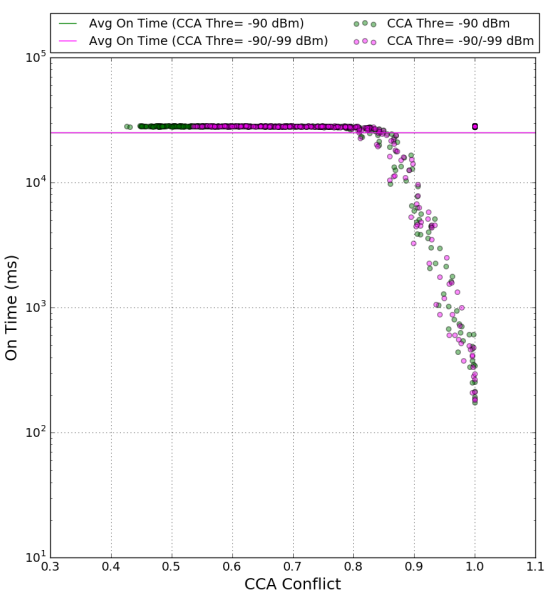

(b) Activity time.

Fig. 5. PSP and Energy Consumption for heterogeneous CCA threshold values in a network with 500 nodes and M=7.

nodes that are getting a low success probability are the ones with a smaller CCA conflict rate. Therefore, we investigate the consequences of reducing the CCA threshold only for these particular nodes, which improves their visibility of formerly hidden terminals, and so their ability to avoid collisions. For this, we changed the CCA threshold for the nodes presenting a CCA conflict rate below 0.6 from $-90 \mathrm{dBm}$ to $-99 \mathrm{dBm}$.

The simulation results of this scenario are shown in Fig. $5 \mathrm{a}$, where we remark a slight increase in packet success rate following this adaptation. However, the important gain is obtained at a local level: all the nodes in the network now show a PSP above $10 \%$. This means that, by simply using two values for the CCA threshold in a network, it is possible to eliminate completely disconnected nodes, which accounted for $5 \%$ of the nodes under homogeneous settings. On the energy consumption side, see Fig. 5b, we do not notice any important change and this can be considered as a good result since we keep consuming the same amount of energy while we get a better local and global PSP result.

\section{CONCLUSION}

With carrier sense mechanisms beginning to be integrated in MAC layer solutions of the IoT world, we investigate the impact of the CCA threshold on the performance of the network. We show that carrier sense mechanisms can bring benefits even if a node is not capable of sensing all its contenders. However, depending on the topology of the network, some nodes sense more transmissions than others, which results in much better performance. Using different CCA thresholds in the network can reduce the impact of this phenomenon, improving the fairness in the network.

\section{ACKNOWLEDGMENT}

This work has been partly supported by the INSA Lyon SPIE ICS chair on the Internet of Things.

\section{REFERENCES}

[1] Y. Mo, C. Goursaud, J.M. Gorce. "Theoretical Analysis of UNB-based IoT Networks with Path Loss and Random Spectrum Access", Proc. IEEE PIMRC 2016, Valencia, Spain, Sep. 2016.

[2] O. Iova, A.L. Murphy, G.P. Picco, L. Ghiro, D. Molteni, F. Ossi, F. Cagnacci, "LoRa from the City to the Mountains: Exploration of Hardware and Environmental Factors", Proc. MadCom 2017, Uppsala, Sweden, Feb. 2017.

[3] E. Wang, X. Lin, A. Adhikary, A. Grovlen, Y. Sui, Y. Blankenship, J. Bergman, H.S. Razaghi, "A Primer on 3GPP Narrowband Internet of Things", IEEE Communications Magazine, vol. 55, no. 3, pp. 117-123, Mar. 2017.

[4] T. Adame, A. Bel, B. Bellalta, J. Barcelo, M. Oliver, "IEEE 802.11ah: The WiFi Approach for M2M Communications", IEEE Wireless Communications, vol. 21, no. 6, pp. 144-152, Dec. 2014.

[5] K. Kosek-Szott. "A Survey of MAC Layer Solutions to the Hidden Node Problem in Ad-Hoc Networks", Ad Hoc Networks, vol. 10, no. 3, pp. 635-660, May 2012.

[6] T.-H. To, A. Duda. "Simulation of LoRa in NS-3: Improving LoRa Performance with CSMA", Proc. IEEE ICC 2018, Kansas City, MO, USA, May 2018.

[7] C. Pham. "Robust CSMA for Long-Range LoRa Transmissions with Image Sensing Devices", Proc. IFIP Wireless Days 2018, Dubai, UAE, Apr. 2018.

[8] E. Haghani, M.N. Krishnan, A. Zakhor. "Adaptive Carrier-Sensing for Throughput Improvement in IEEE 802.11 Networks", Proc. IEEE Globecom 2010, Miami, FL, USA, Dec. 2010.

[9] X. Yang, N. Vaidya. "On Physical Carrier Sensing in Wireless Ad Hoc Networks", Proc. IEEE Infocom 2005, Miami, FL, USA, Mar. 2005

[10] R. Stanica, E. Chaput, A.-L. Beylot. "Physical Carrier Sense in Vehicular Ad-Hoc Networks", Proc. IEEE MASS 2011, Valencia, Spain, Oct. 2011.

[11] E. Khorov, A. Lyakhov, A. Krotov, A. Guschin. "A Survey on IEEE 802.11ah: An Enabling Networking Technology for Smart Cities", Computer Communications, vol. 58, no. 1, pp. 53-69, Mar. 2015.

[12] M.S. Afaqui, E. Garcia-Villegas, E. Lopez-Aguilera, G. Smith, D. Camps. "Evaluation of Dynamic Sensitivity Control Algorithm for IEEE 802.11ax", Proc. IEEE WCNC 2015, New Orleans, LA, USA, Mar. 2015.

[13] A. Ben Khalifa, R. Stanica. "Performance Evaluation of Channel Access Methods for Dedicated IoT Networks", Proc. IFIP Wireless Days 2018, Manchester, UK, Apr. 2019.

[14] Z. Zeng, Y. Yang, J.C. Hou. "How Physical Carrier Sense Affects System Throughput in IEEE 802.11 Wireless Networks", Proc. IEEE Infocom 2008, Phoenix, AZ, USA, Apr. 2008.

[15] ns-3 Network Simulator (online) https://www.nsnam.org/

[16] L.B. Jiang, S.C. Liew. "Improving Throughput and Fairness by Reducing Exposed and Hidden Nodes in 802.11 Networks", IEEE Transactions on Mobile Computing, vol. 7, no. 1, pp. 34-49, Jan. 2008. 